OPEN ACCESS

Edited by:

Hong-Qi Zhang,

Capital Medical University, China

Reviewed by:

Tao Hong,

Capital Medical University, China Waldo Rigoberto Guerrero, University of South Florida,

United States

*Correspondence:

Jinlu Yu

jlyu@jlu.edu.cn

orcid.org/0000-0003-2329-7946

Specialty section:

This article was submitted to Endovascular and Interventional Neurology,

a section of the journal Frontiers in Neurology

Received: 03 March 2021 Accepted: 12 May 2021

Published: 31 May 2021

Citation:

Su H, Xu K, Wang Y and Yu J (2021) Is the Middle Meningeal Artery the Optimal Path for Dural Arteriovenous

Fistula Embolization?

Front. Neurol. 12:675355 doi: 10.3389/fneur.2021.675355

\section{Is the Middle Meningeal Artery the Optimal Path for Dural Arteriovenous Fistula Embolization?}

\author{
Han Su, Kan Xu, Yiheng Wang and Jinlu Yu* \\ Department of Neurosurgery, The First Hospital of Jilin University, Changchun, China
}

Background: The middle meningeal artery (MMA) is the optimal arterial path for endovascular treatment (EVT) of dural arteriovenous fistulas (DAVFs). However, the details are not completely understood.

Materials and Methods: We performed a retrospective study of patients who were admitted to the First Hospital of Jilin University with a diagnosis of cranial DAVF with involvement of the MMA as a feeding artery. On the basis of the arterial path chosen and the role of the MMA in the first EVT procedure, EVT was divided into three types $(I-I I)$, each of which was further divided into two subclasses (a and b). The degree of embolization was analyzed.

Result: The 104 included patients ranged in age from 13 to 80 years (mean, $53.6 \pm 11.8$ years). There were 48 cases of hemorrhage (46.2\%, 48/104). Complete embolization was achieved in the first procedure in $64.4 \%$ of cases, and success was eventually achieved using EVT (the first attempt or a subsequent attempt) in $74.1 \%$ of cases. EVT caused complications in $6.7 \%$ of cases. A modified Rankin scale score of 0 or 1 was achieved in $78.8 \%$ of patients. Statistical analyses revealed that type la and Ilb EVTs had the lowest complete embolization rates, but no difference was found between type la and Ilb EVTs. Types Ila and III EVT had the highest complete embolization rates. Most cases had a good prognosis.

Conclusion: These findings elucidate the features of the different EVT classes defined by the first EVT procedure and the role of the MMA. The delivery of treatment via slim and tortuous MMA branches increased the failure rate of EVT. A thick, straight MMA branch is the optimal path for treatment.

Keywords: middle meningeal artery, dural arteriovenous fistula, endovascular treatment, classification, prognosis

\section{INTRODUCTION}

Cranial dural arteriovenous fistula (DAVF) is a rare condition that involves a direct arteriovenous connection within the dural leaflets. This condition accounts for a small proportion, $\sim 10-15 \%$, of all intracranial arteriovenous shunts (1). The clinical symptoms of DAVFs depend on the draining vein involved (Figure 1). DAVFs recruit many arteries to act as feeders; although the external carotid artery is usually involved, the meningeal branch of the internal carotid artery, the dural branches of the intracranial arteries, and the posterior meningeal artery (PMA, most commonly arising from the vertebral artery) may be involved (2). 
Of all DAVF feeding arteries, the middle meningeal artery (MMA) of the external carotid artery is the most important because of its wide range of involvement, and all branches of the MMA can feed DAVFs (Figure 2). Endovascular treatment $(\mathrm{EVT})$ is the primary treatment for DAVFs and focuses on the MMA (3). The first EVT procedure via the MMA determines its success or failure (4). Nevertheless, although the MMA is the gold standard arterial path of EVT for DAVFs, many factors influence the success rate (5).
A

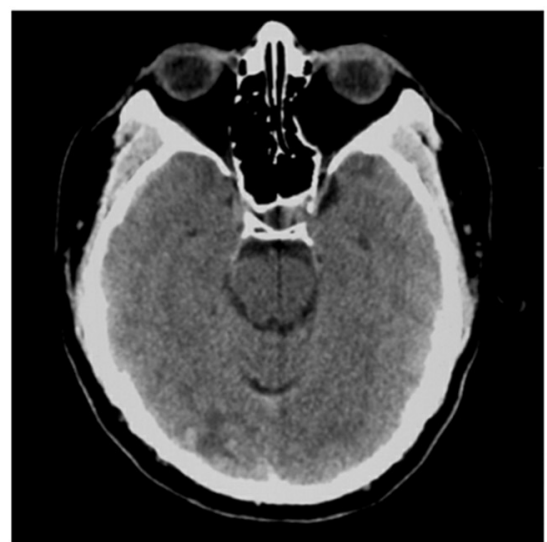

C

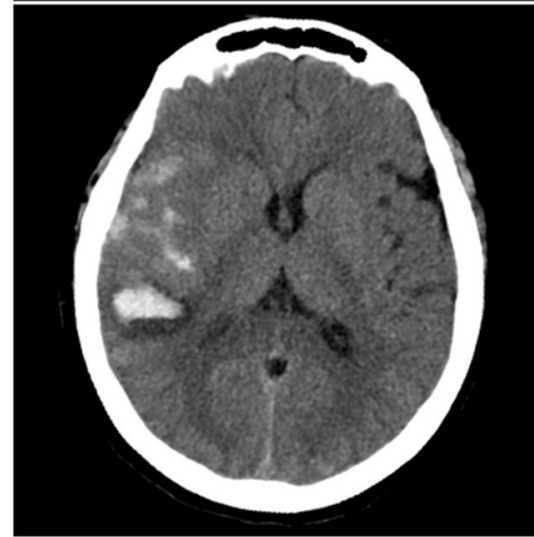

E

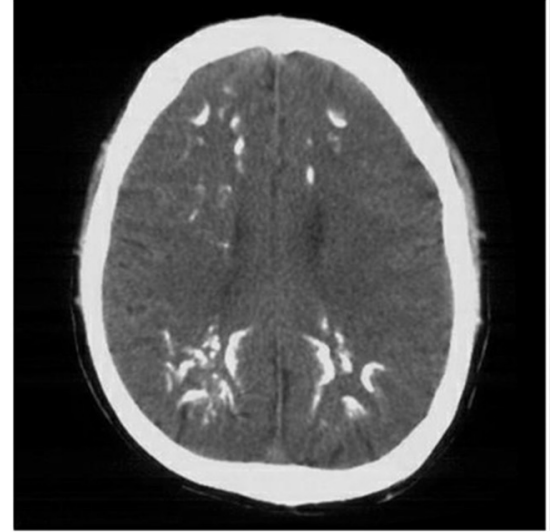

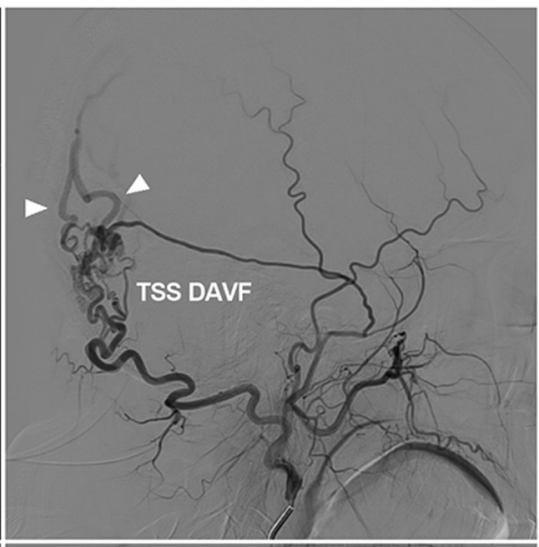

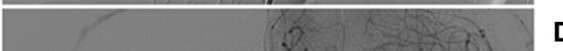

D

B
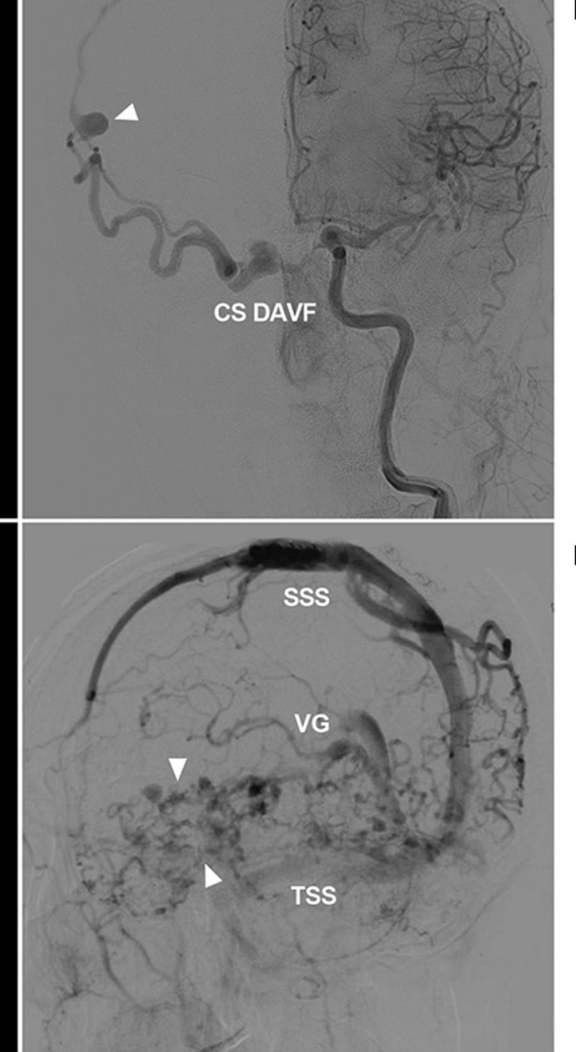

FIGURE 1 | Venous drainage patterns of DAVFs. (A,B) Unruptured DAVF with images from the same patient. CT (A) showed a mix of high and low densities in the right occipital lobe. DSA (B) showed a TSS DAVF draining to multiple cortical veins (triangles), and the veins throughout the entire brain were normal and not dilated. (C,D) Hemorrhagic DAVF with images from the same patient. CT (C) showed subarachnoid hemorrhage and hematoma in the Sylvian fissure region. DSA (D) showed a CS DAVF with cortical vein drainage and a ruptured venous aneurysm (triangle). (E,F) Extensive pseudophlebitic DAVF with images from the same patient. CT (E) showed multiple calcifications in the white matter around the ventricle. DSA (F) showed an extensive pseudophlebitic pattern involving superficial and deep veins (triangles). CS, cavernous sinus; CT, computed tomography; DAVF, dural arteriovenous fistula; DSA, digital subtraction angiography; SSS, superior sagittal sinus; TSS, transverse-sigmoid sinus; VG, vein of Galen. 
A

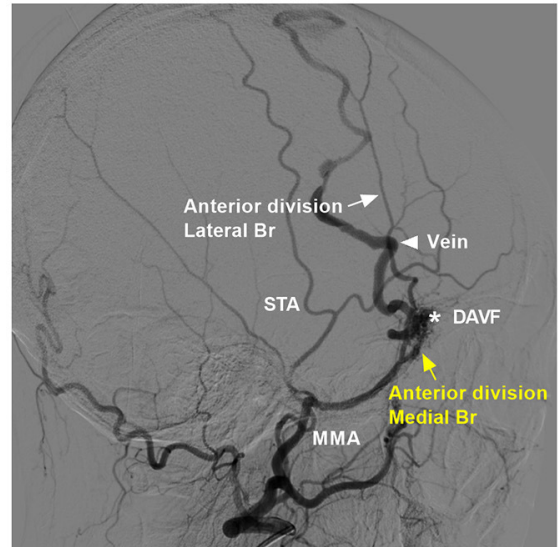

C

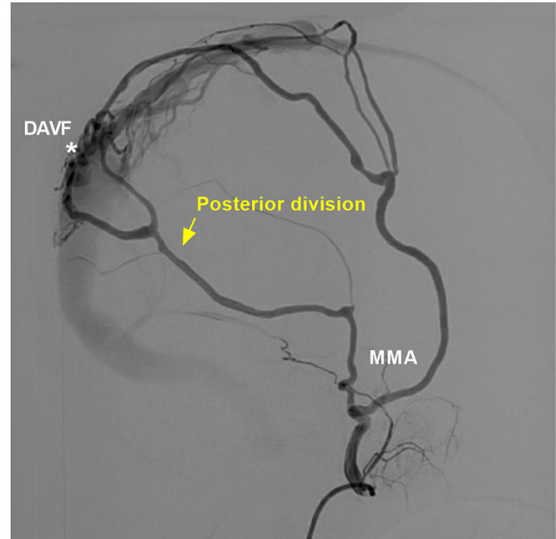

E

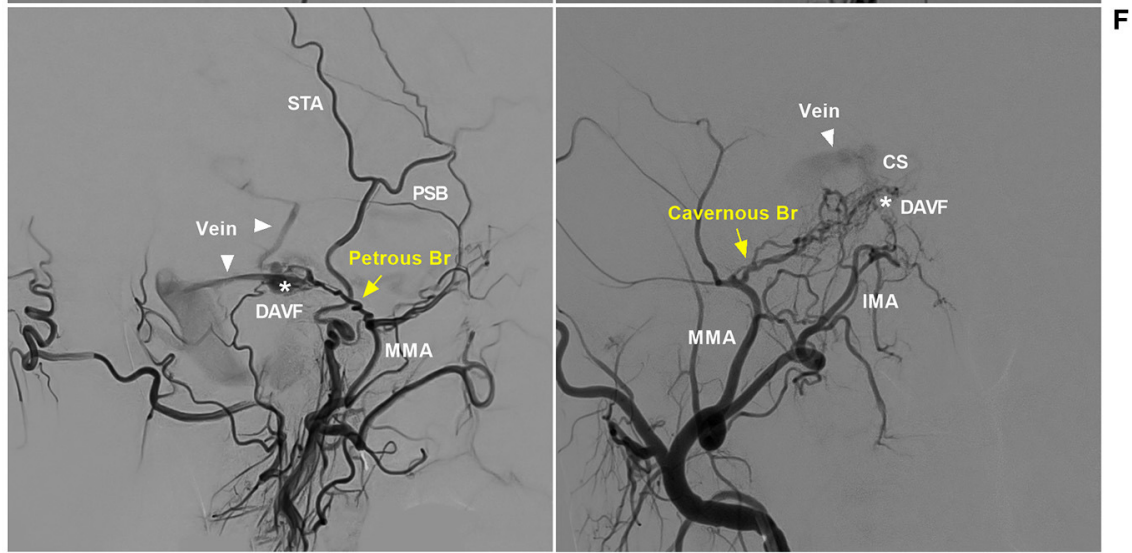

B

D

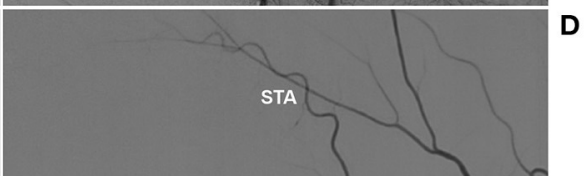

FIGURE 2 | Pathological anatomy of the MMA as a DAVF feeder. (A) DSA of the lateral view showed the sphenoid wing DAVF (asterisk) fed by the medial branch (sphenoid) of the anterior division of the MMA (yellow arrow) draining to the SSS (triangle). (B) DSA of the lateral view showed a DAVF (asterisk) at the anterior falx cerebri fed by the lateral branch of the anterior division of the MMA (yellow arrow) that reached the midline and anastomosed with the AFA (yellow arrow). The DAVF drained through the cortical vein (triangle) to the SSS. (C) DSA of the lateral view showed that the posterior division of the MMA supplied the DAVF (asterisk) at the SSS. (D) DSA of the lateral view showed that the PSB of the MMA (yellow arrow) supplied the TSS DAVF (asterisk). (E) DSA of the lateral view showed that the PB of the MMA (yellow arrow) supplied the tentorium DAVF (asterisk), and the triangles indicate the draining veins. (F) DSA of the anterior posterior view showed the CS DAVF (asterisk) supplied by the cavernous branch, and the triangle indicates the draining vein. AFA, anterior falx artery; Br, branch; CS, cavernous sinus; DAVF, dural arteriovenous fistula; DSA, digital subtraction angiography; IMA, internal maxillary artery; MMA, middle meningeal artery; PB, petrous branch; PSB, petrosquamosal branch; SSS, superior sagittal sinus; STA, superficial temporal artery; TSS, transverse-sigmoid sinus.

Few studies have focused on the role of the MMA in EVT of DAVFs. Therefore, we performed a retrospective single-center investigation of patients who were diagnosed with a DAVF with MMA involvement.

\section{MATERIALS AND METHODS}

Patients who were admitted to the First Hospital of Jilin University and diagnosed with a cranial DAVF with MMA 

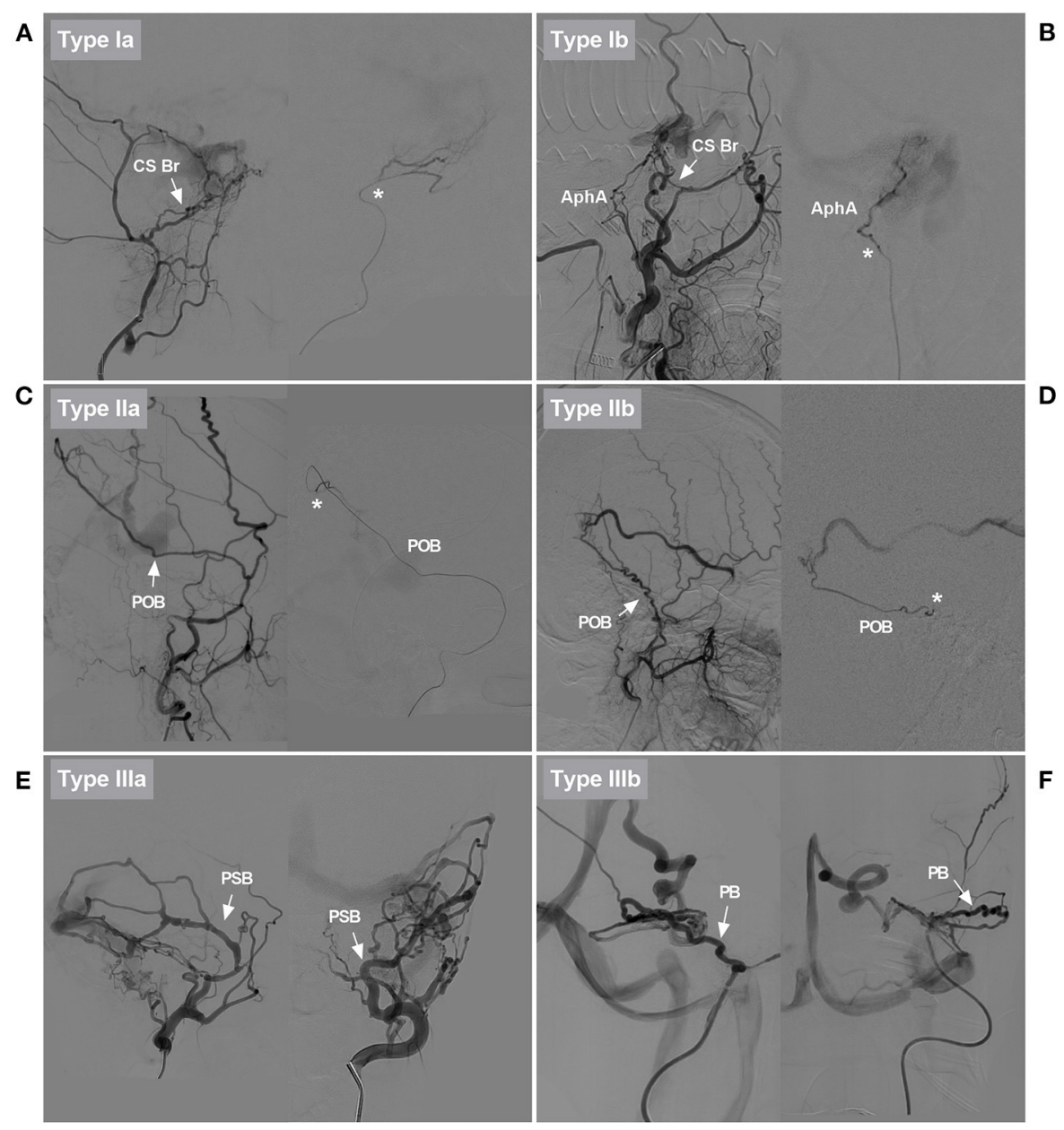

FIGURE 3 | Classification of EVTs based on the role of the MMA. (A) DSA showed that the CS branch of the MMA in type la EVT was slim and tortuous (arrow). Superselective angiography showed the CS branch (asterisk). EVT was delivered via that vessel. (B) DSA showed that the CS branch of the MMA in type Ib EVT was slim and tortuous (arrow). EVT was delivered via the AphA, which was thicker than the CS branch. AphA was shown on superselective angiography (asterisk). (C) DSA showed that the POB of the MMA in type lla EVT was normally developed, sufficiently thick, and straight (arrow). Superselective angiography showed the POB (asterisk). EVT was delivered via that vessel. (D) DSA showed that the PSB of the MMA in type llb EVT was normally developed, but it was tortuous. Superselective angiography showed the PSB (asterisk). Because there was no other suitable artery, the PSB was used to deliver EVT. (E) Different DSA views showed that the PSB of the MMA in type IIla EVT was overdeveloped and straight (arrows). EVT was delivered via that vessel. (F) Different DSA views showed that the PB of the MMA in type IIIb EVT was overdeveloped and tortuous (arrows). EVT was delivered via that vessel. AphA, ascending pharyngeal artery; Br, branch; CS, cavernous sinus; DAVF, dural arteriovenous fistula; DSA, digital subtraction angiography; EVT, endovascular treatment; MMA, middle meningeal artery; PB, petrous branch; POB,

parieto-occipital branch; PSB, petrosquamosal branch.

involvement as a feeding artery from October 2012 to October 2020 were included in this retrospective study. The institutional ethics committee approved this study.

\section{Inclusion and Exclusion Criteria}

The inclusion criteria were as follows: (1) cranial DAVFs with feeding arteries from the MMA alone or in combination with other arteries; (2) DAVFs treated with EVT via the MMA or other arteries; and (3) no previous EVT, open surgery, or radiosurgery performed before admission to our institution. Patients with DAVFs treated with transvenous EVT were excluded.

\section{Strategy and Process of EVT}

For cranial DAVFs with MMA as the feeding artery, digital subtraction angiography (DSA) was performed to assess the angioarchitecture of the DAVF, and the feeding artery, fistula size, draining vein, location, Cognard grade, and other parameters were recorded. When performing EVT, a Marathon or Apollo microcatheter (Medtronic, Irvine, California, USA) was used to approach the DAVF as closely as possible, and Onyx (Medtronic, Irvine, California, USA) was injected to cast the DAVF as much as possible in order to penetrate the draining vein. The EVT procedure was monitored simultaneously using angiography of another artery when necessary. If the DAVF could not be seen via 


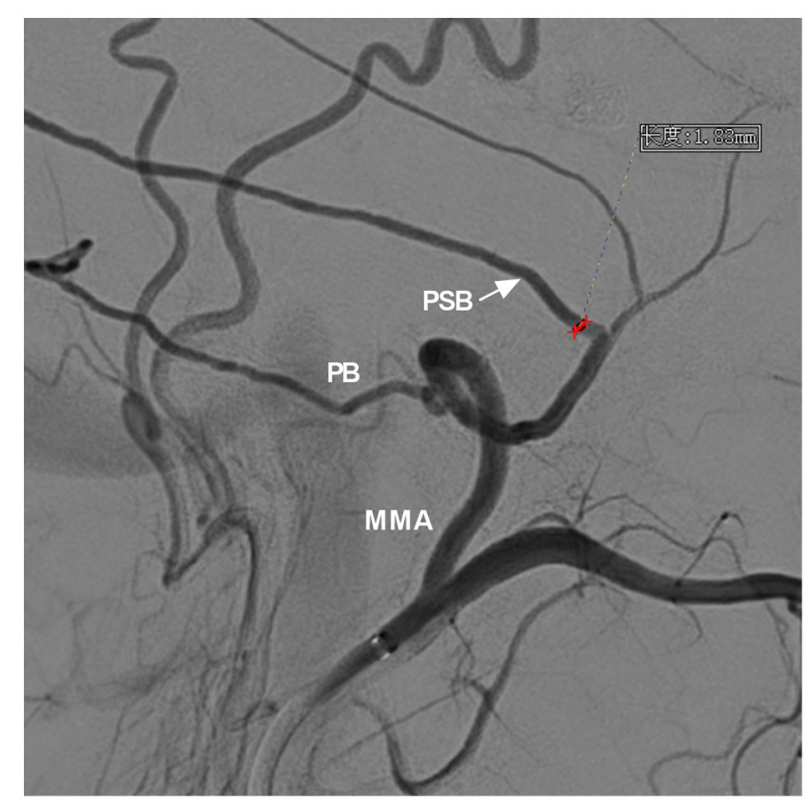

FIGURE 4 | Measurement of the diameter of the MMA branch origin from the trunk. DSA showed the MMA and its branches. For the case of a DAVF with the PSB and PB acting as the main feeding arteries, the PSB (arrow) was chosen as the arterial path to perform EVT, and the diameter of the PSB origin from the trunk was measured (red line segment). DSA, digital subtraction angiography; EVT, endovascular treatment; MMA, middle meningeal artery; PB, petrous branch; PSB, petrosquamosal branch.

the MMA and other feeding arteries, complete embolization was achieved or the EVT was incomplete.

\section{Classification of EVT}

The present study divided EVT into three types and six subclasses based on the arterial path chosen in the first EVT procedure and the role of the MMA in EVT.

Type I EVT: The MMA as the feeding artery was slim and tortuous, and the EVT was further divided into types a and b. In type Ia EVT, the slim and tortuous MMA branch was used to perform the embolization (Figure 3A). In type Ib EVT, a better-suited artery than the MMA was used to perform the embolization (Figure 3B).

Type II EVT: The MMA branch chosen to perform the EVT was neither slim nor normally developed, and the EVT was further divided into types a and b. In type IIa EVT, the straight MMA was used to perform the embolization (Figure 3C). In type IIb EVT, the tortuous MMA branch was used to perform the embolization (Figure 3D).

Type III EVT: The MMA branch chosen to perform EVT was overdeveloped and hyperplastic, and the EVT was further divided into types a and b. In type IIIa EVT, the straight MMA branch was used to perform the embolization (Figure 3E). In type IIIb EVT, the tortuous MMA branch was used to perform the embolization (Figure 3F).

The diameter of the MMA branch originating from the trunk as the arterial path was measured in these types (Figure 4).

\section{Evaluation of Short-Term and Follow-Up Outcomes}

EVT complications and resolutions, the length of hospital stay and the modified Rankin Scale (mRS) score at 3-6 months and long-term follow-up were recorded.

\section{Statistical Analysis}

Statistical analyses were performed using GraphPad software (LLC, San Diego, CA, USA). Continuous variables are expressed as the means \pm standard deviation. The chi-squared test was used to analyze count data. $P<0.05$ was considered statistically significant.

\section{RESULTS}

\section{General Information}

The 104 patients were aged 13 to 80 years (mean, $53.6 \pm$ 11.8 years) and included 42 females $(40.4 \%, 42 / 104)$ and 62 males $(59.6 \%, 62 / 104)$. There were 56 cases of unruptured DAVFs $(53.8 \%, 56 / 104)$, including 20 cases with headache and dizziness, five cases with tinnitus and intracranial murmur, seven cases with physical manifestations, 15 cases with ocular symptoms (exophthalmos and chemosis), and nine cases with cognitive deficiencies.

There were 48 cases of hemorrhage (46.2\%, 48/104), including 19 cases of subarachnoid hemorrhage (SAH), 11 cases of SAH in combination with intracerebral hematoma (IH) and/or intraventricular hemorrhage (IVH), 13 cases of IH, and five cases of IH and IVH. Among the 48 cases, 17 cases were Hunt-Hess grade I, 22 cases were grade II, and nine cases were grade III.

\section{Imaging Characteristics \\ DAVF Location and Size \\ DAVF Location}

The most common location was the transverse-sigmoid sinus (TSS) $(31.7 \%)$, followed by the tentorium $(21.2 \%)$, cavernous sinus (12.5\%), and superior sagittal sinus (10.6\%). Table 1 shows the detailed data.

\section{DAVF Size}

Except for six diffuse and extensive DAVFs that could not be measured, the sizes of the other 98 DAVFs were between 0.5 and $6.5 \mathrm{~cm}$ (mean, $3.1 \pm 1.1 \mathrm{~cm}$ ).

\section{Distribution of the Feeding Artery}

The feeding arteries of all 104 DAVFs included the MMA. The ipsilateral MMA was involved in 77 DAVFs $(74.0 \%, 77 / 104)$, and the bilateral MMAs were involved in 27 DAVFs $(26.0 \%, 27 / 104)$. The other feeding arteries were the occipital artery (OA) $(47.1 \%)$, PMA (29.8\%), and meningohypophyseal trunk (MHT) (26.0\%). Table 2 shows the detailed data.

\section{Venous Drainage Pattern}

Multiple cortical veins providing drainage were the most common pattern in the DAVFs (33.7\%), followed by a single cortical vein $(19.2 \%)$, a single deep vein $(9.6 \%)$, multiple deep veins $(9.6 \%)$, and the vein of Galen with reverse flow $(4.8 \%)$. Table 3 shows the detailed data. 
TABLE 1 | Locations of dural arteriovenous fistulas.

\begin{tabular}{lcc}
\hline Location & Number & Percentage \\
\hline Transverse-sigmoid sinus & 33 & $31.7 \%(33 / 104)$ \\
Tentorium & 22 & $21.2 \%(22 / 104)$ \\
Cavernous sinus & 13 & $12.5 \%(13 / 104)$ \\
Superior sagittal sinus & 11 & $10.6 \%(11 / 104)$ \\
Anterior cranial fossa & 7 & $6.7 \%(7 / 104)$ \\
Sphenoid wing & 6 & $5.8 \%(6 / 104)$ \\
Posterior falx cerebri & 2 & $1.9 \%(2 / 104)$ \\
Anterior falx cerebri & 2 & $1.9 \%(2 / 104)$ \\
Temporal region & 2 & $1.9 \%(2 / 104)$ \\
Torcular Herophili & 2 & $1.9 \%(2 / 104)$ \\
Middle cranial fossa & 1 & $1.0 \%(1 / 104)$ \\
Occipital region & 1 & $1.0 \%(1 / 104)$ \\
Parietal region & 1 & $1.0 \%(1 / 104)$ \\
Frontal region & 1 & $1.0 \%(1 / 104)$ \\
Total & 104 & $100 \%(104 / 104)$
\end{tabular}

TABLE 2 | Distribution of feeding arteries of dural arteriovenous fistulas.

\begin{tabular}{lcc}
\hline Artery & Number & Percentage \\
\hline Middle meningeal artery & 104 & $100 \%(104 / 104)$ \\
Occipital artery & 49 & $47.1 \%(49 / 104)$ \\
Posterior meningeal artery & 31 & $29.8 \%(31 / 104)$ \\
Meningohypophyseal trunk & 27 & $26.0 \%(27 / 104)$ \\
Inferior lateral trunk & 12 & $11.5 \%(12 / 104)$ \\
Ascending pharyngeal artery & 12 & $11.5 \%(12 / 104)$ \\
Ophthalmic artery & 10 & $9.6 \%(10 / 104)$ \\
Posterior cerebral artery & 9 & $8.7 \%(9 / 104)$ \\
Accessory meningeal artery & 6 & $5.8 \%(6 / 104)$ \\
Superficial temporal artery & 5 & $4.8 \%(5 / 104)$ \\
Middle cerebral artery & 5 & $4.8 \%(5 / 104)$ \\
Anterior inferior cerebellar artery & 3 & $2.9 \%(3 / 104)$ \\
Superior cerebellar artery & 3 & $2.9 \%(3 / 104)$ \\
\hline
\end{tabular}

TABLE 3 | Venous drainage patterns of dural arteriovenous fistulas.

\begin{tabular}{lcc}
\hline Draining vein & Number & Percentage \\
\hline Multiple cortical veins & 35 & $33.7 \%(7 / 104)$ \\
Single cortical vein & 20 & $19.2 \%(20 / 104)$ \\
Venous sinus or ophthalmic vein & 13 & $12.5 \%(14 / 104)$ \\
Single deep vein & 10 & $9.6 \%(10 / 104)$ \\
Multiple deep veins & 10 & $9.6 \%(10 / 104)$ \\
Both cortical and deep veins & 7 & $6.7 \%(7 / 104)$ \\
Vein of Galen with reverse flow & 5 & $4.8 \%(5 / 104)$ \\
Spinal cord vein & 4 & $3.8 \%(4 / 104)$ \\
Total & 104 & $100 \%(104 / 104)$
\end{tabular}

\section{Cognard Grade of DAVFs}

Four of the 104 DAVFs were Cognard grade I $(3.8 \%, 4 / 104)$, five DAVFs were grade IIa $(4.8 \%, 5 / 104)$, two DAVFs were grade IIb
TABLE 4 | Baseline data of type I, II, and III EVTs.

\begin{tabular}{lccccl}
\hline & I $(\boldsymbol{n}=\mathbf{3 0})$ & II $(\boldsymbol{n}=\mathbf{5 7})$ & $\mathbf{I I I}(\boldsymbol{n}=\mathbf{1 7})$ & $\boldsymbol{P}$-value \\
\hline Age (years) & $59.1 \pm 9.1$ & $52.0 \pm 12.3$ & $49.4 \pm 11.5$ & $\begin{array}{l}0.2146 \\
\text { (Bartlett's test) }\end{array}$ \\
Sex (male) & 16 & 34 & 12 & $\begin{array}{l}0.5112 \\
\text { (Chi-square, 1.342) }\end{array}$ \\
$\begin{array}{l}\text { Nonhemorrhagic } \\
\text { onset }\end{array}$ & 15 & 31 & 10 & $\begin{array}{l}0.8375 \\
\text { (Chi-square, 0.356) }\end{array}$
\end{tabular}

EVT, endovascular treatment.

TABLE 5 | Arterial path used in the first EVT procedure.

\begin{tabular}{lcc}
\hline Arterial path & Number & Percentage \\
\hline Petrosquamosal branch & 36 & $34.6 \%(36 / 104)$ \\
Parieto-occipital branch & 15 & $14.4 \%(15 / 104)$ \\
Petrous branch & 10 & $9.6 \%(10 / 104)$ \\
Cavernous sinus branch & 11 & $10.6 \%(11 / 104)$ \\
Medial branch of anterior division & 7 & $6.7 \%(7 / 104)$ \\
Lateral branch of anterior division & 6 & $5.8 \%(6 / 104)$ \\
Accessory meningeal artery & 3 & $2.9 \%(3 / 104)$ \\
Occipital artery & 9 & $8.5 \%(9 / 104)$ \\
Ascending pharyngeal artery & 3 & $2.9 \%(3 / 104)$ \\
Ophthalmic artery & 2 & $1.9 \%(2 / 104)$ \\
Internal maxillary artery & 2 & $1.9 \%(2 / 104)$ \\
Total & 104 & $100 \%(104 / 104)$
\end{tabular}

EVT, endovascular treatment.

(1.9\%, 2/104), 22 DAVFs were grade IIa $+\mathrm{b}(21.2 \%, 22 / 104), 10$ DAVFs were grade III $(9.6 \%, 10 / 104), 57$ DAVFs were grade IV $(54.8 \%, 57 / 104)$, and four DAVFs were grade V $(3.8 \%, 4 / 104)$.

\section{EVT Classification and Choice of the Arterial Path EVT Classification}

Based on the arterial path chosen in the first EVT procedure, type Ia EVT was performed for 14 DAVFs $(13.5 \%, 14 / 104)$, type Ib EVT was performed for 16 DAVFs $(15.4 \%, 16 / 104)$, type IIa EVT was performed for 48 DAVFs (46.1\%, 48/104), type IIb EVT was performed for nine DAVFs (8.7\%, 9/104), type IIIa EVT was performed for 11 DAVFs $(10.6 \%, 11 / 104)$, and type IIIb EVT was performed for six DAVFs $(5.8 \%, 6 / 104)$.

The baseline data of patients were compared among types I, II, and III EVT. There was no difference in age, sex, or onset (Table 4).

\section{Choice of the Arterial Path}

Among the 104 first EVT procedures, MMA branches were used in 88 of them $(84.6 \%, 88 / 104)$, most commonly the petrosquamosal branch (PSB) (34.6\%), followed by the parietooccipital branch (POB) (14.4\%), cavernous sinus branch (10.6\%), and petrous branch (9.6\%). Type Ib EVT was performed via the $\mathrm{OA}$ in 16 cases $(8.5 \%)$, followed by the ascending pharyngeal artery (AphA) (2.9\%) and ophthalmic artery (OphA) (1.9\%). Table 5 shows the detailed data. 
TABLE 6 | Baseline data in patients with incomplete and complete EVTs.

\begin{tabular}{|c|c|c|c|}
\hline & $\begin{array}{l}\text { Incomplete EVT } \\
\qquad(n=37)\end{array}$ & $\begin{array}{l}\text { Complete EVT } \\
\qquad(n=67)\end{array}$ & $P$-value \\
\hline Age (years) & $54.7 \pm 12.7$ & $53.1 \pm 11.3$ & $\begin{array}{l}0.5172 \text { (Unpaired } \\
t \text {-test) }\end{array}$ \\
\hline Sex (male) & 18 & 44 & $\begin{array}{l}0.0998 \text { (Fisher's } \\
\text { exact test) }\end{array}$ \\
\hline Nonhemorrhagic & 25 & 31 & $\begin{array}{l}0.0631 \text { (Fisher's } \\
\text { exact test) }\end{array}$ \\
\hline
\end{tabular}

EVT, endovascular treatment.

The diameter of the MMA branch originating from the trunk as the arterial path was obtained only in a few cases: type I $(0.5 \pm$ $0.1 \mathrm{~mm}, n=16)$; type II ( $1.8 \pm 0.2 \mathrm{~mm}, n=26)$; and type III (2.7 $\pm 0.4 \mathrm{~mm}, n=8)$.

\section{EVT Result and Subsequent Processing}

Complete embolization was obtained in 67 cases after the first EVT procedure $(64.4 \%, 67 / 104)$, and incomplete embolization was obtained in 37 cases $(35.6 \%, 37 / 104)$. Among the 37 cases of unsuccessful EVT, Onyx did not reach the fistula point in two cases, and it reached the fistula but did not embolize the DAVF completely in 35 cases.

Among the 37 cases of unsuccessful EVT, temporary conservative treatment was used in 17 cases, and direct surgical resection was performed in four cases. EVT via other arteries was performed in the other 16 cases. Complete EVT was achieved in 10 cases, and conservative treatment was administered in the other six cases.

In summary, the first EVT procedure achieved complete embolization with a success rate of $64.4 \%$ (67/104), and the success rate for the first and subsequent EVT procedures was 74.1\% (77/104).

The baseline data were compared between the incomplete and complete EVT groups, and there was no difference in age, sex, or onset (Table 6).

\section{Complications and Solutions}

EVT resulted in complications in seven cases (6.7\%, 7/104). Facial numbness was observed in two cases, and conservative treatment was administered. Intraoperative or postoperative intracranial hemorrhage occurred in three cases. Hematoma evacuation was applied in two cases, and conservative treatment was administered in one case. Blindness occurred in one case, and conservative treatment was administered. Postoperative hydrocephalus occurred in one case, and a ventriculoperitoneal shunt was performed.

\section{Follow-Up and Outcomes}

The length of hospital stay ranged from 1 to 20 days $(4.2 \pm$ 3.5 days).

The short-term follow-up period ranged from 3 to 6 months. The mRS scores were $0,1,2$, and $3-5$ in 56 (53.8\%, 56/104), 26 $(25 \%, 26 / 104), 18(17.3 \%, 18 / 104)$, and $4(3.8 \%, 4 / 104)$ patients,
TABLE 7 | Statistical analyses of EVT completion.

\begin{tabular}{lcccl}
\hline EVT type & Complete EVT & Incomplete EVT & Total & \\
\hline la & $2(14.3 \%)$ & $12(85.7 \%)$ & 14 & $\begin{array}{l}\text { Chi-squared, 35.4, } \\
P<0.0001\end{array}$ \\
Ib & $10(62.5 \%)$ & $6(37.5 \%)$ & 16 & \\
Illa & $40(87.0 \%)$ & $6(13.0 \%)$ & 46 & \\
Ilb & $3(33.3 \%)$ & $6(66.7 \%)$ & 9 & \\
Illa & $6(85.7 \%)$ & $1(14.3 \%)$ & 7 & \\
Illb & $6(100 \%)$ & $0(0 \%)$ & 6 & \\
Total & $67(68.4 \%)$ & $31(31.6 \%)$ & 98 & \\
\hline
\end{tabular}

Chi-squared $=35.4, P<0.01$, indicating a difference between the six subgroups. EVT, endovascular treatment.

TABLE 8 | Statistical comparisons between type la or llb EVTs and other EVTs.

\begin{tabular}{lcc}
\hline EVT type & la & Ilb \\
\hline la & NA & $P=0.3428$ \\
Ib & $P=0.0106$ & $P=0.2262$ \\
Ila & $P<0.0001$ & $P=0.0019$ \\
Ilb & $P=0.3428$ & NA \\
III & $P<0.0001$ & $P=0.0066$ \\
\hline
\end{tabular}

Fisher's exact test was performed, and the analysis showed that type la and IIb EVTs had the lowest complete embolization rates but that there was no difference between type la and IIb EVTs. Types Ila and III EVT had the highest complete embolization rates. EVT, endovascular treatment. NA, not applicable.

respectively. Good short-term recovery (mRS score of 0 or 1 ) was achieved in $78.8 \%(82 / 104)$ of the patients.

The long-term follow-up period ranged from 9 to 102 months. All 59 patients with telephone follow-up data had a mRS score of 0 or 1 . Only 31 patients had angiographic follow-up data. Twenty-two patients had no recurrence, and nine patients had incomplete embolization and accepted retreatment.

\section{Statistical Analysis}

The relationship between the EVT degree and the artery chosen as the path of the first EVT procedure was analyzed. After excluding six diffuse and extensive DAVFs, 98 DAVFs with first EVT attempts were included in the statistical analysis.

The chi-squared test was used to identify differences between multiple groups. Types Ia and IIb EVTs had the lowest complete embolization rates, but there was no difference between types Ia and IIb EVTs. Types IIa and III had the highest complete embolization rates. Tables 7,8 show the detailed data.

We also demonstrated some typical and educational cases of EVT (Figures 5-9).

\section{DISCUSSION}

A DAVF is an arteriovenous shunt located in the dura. The current treatment is EVT, which can involve transarterial and transvenous approaches (6). Although the transvenous approach 


\section{A \\ C}
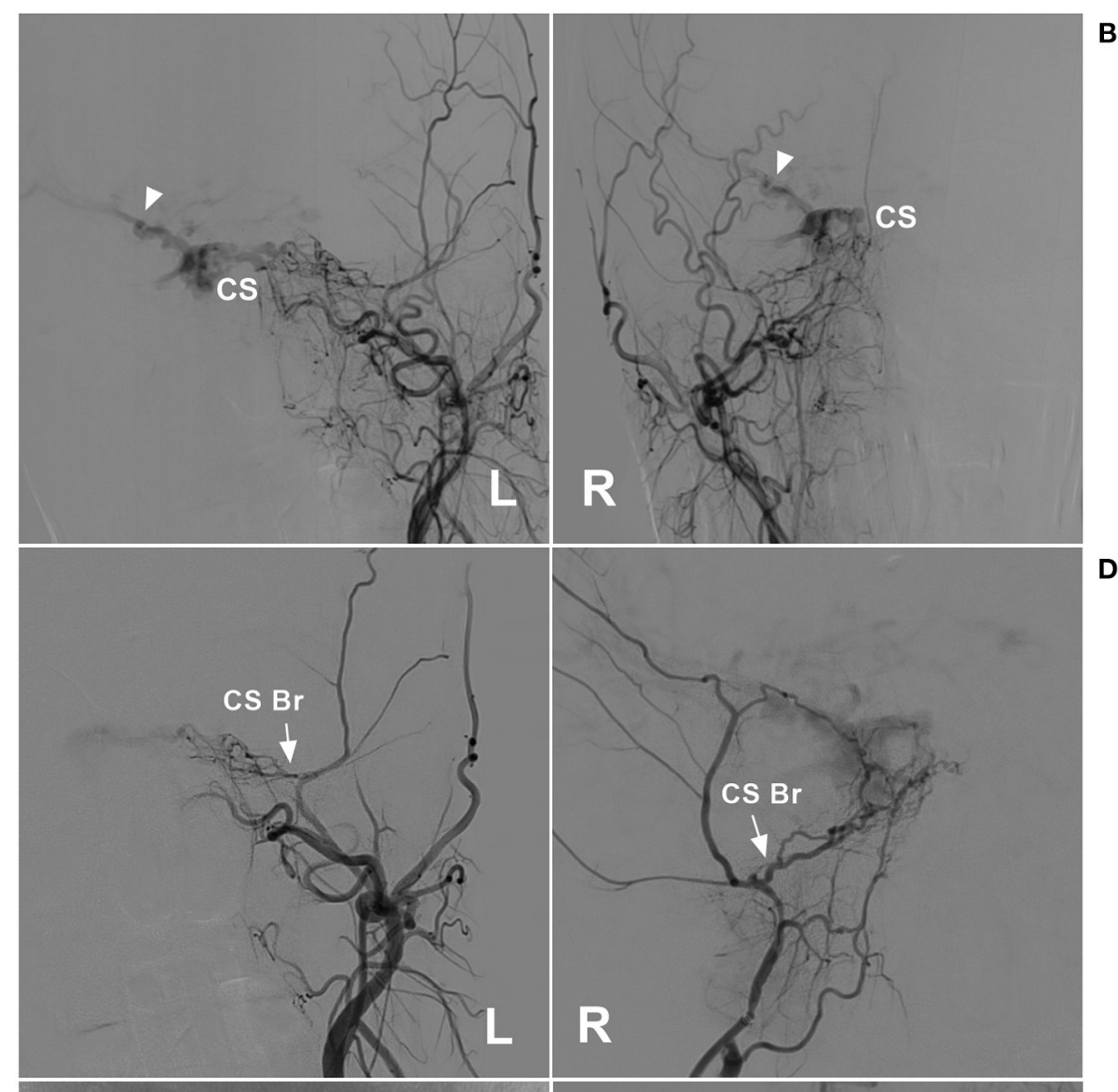

E

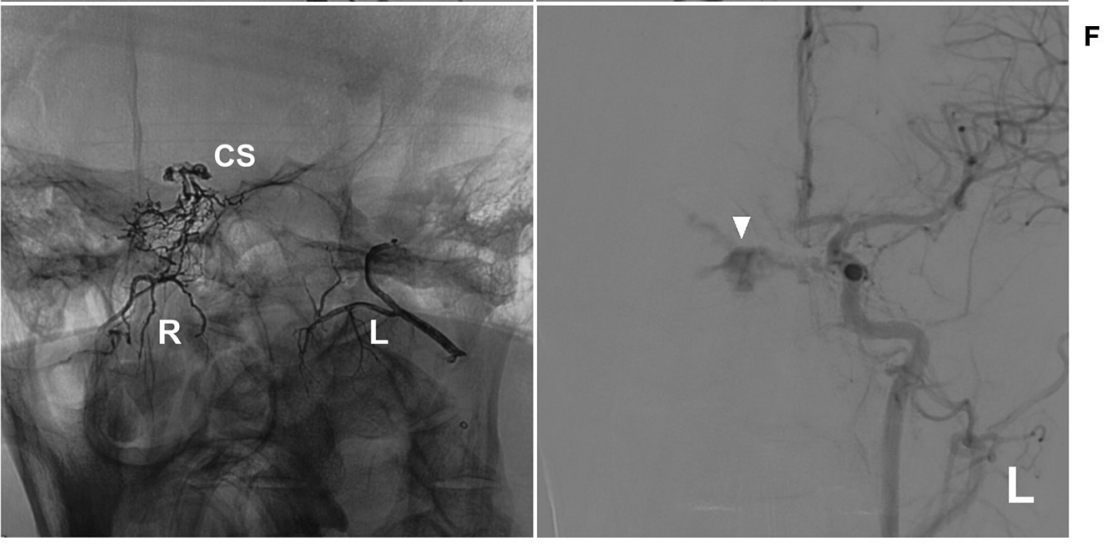

FIGURE 5 | Unsuccessful type la EVT of a CS DAVF. (A,B): Left (A) and right (B) DSA of the anterior posterior view showed a CS DAVF with cortical vein drainage in the Sylvian fissure (triangles). (C,D) Left (C) and right (D) DSA showed the CS branch supplying the DAVF (arrows). (E) X-ray film showed bilateral embolization with Onyx. Onyx was successfully injected into the CS. (F) DSA of the left carotid artery showed that the EVT was incomplete, and the DAVF remained visible (triangle). Br, branch; CS, cavernous sinus; DAVF, dural arteriovenous fistula; DSA, digital subtraction angiography; EVT, endovascular treatment; L, left; R, right.

is an effective method for treating some DAVFs, such as EVT via the cavernous sinus and certain TSSs, the transarterial approach remains the primary approach for treating most DAVFs (7).

Most of the feeding arteries may be used for the arterial path, but the MMA always plays the most important role. Indeed, the MMA is the gold standard artery for the transarterial approach for DAVFs (8). However, few studies have examined the role and influencing factors of the MMA in EVT. Our study collected data from 104 DAVFs with MMA involvement as the feeding artery for analysis of the treatment success rate.

The MMA is widely distributed throughout the cranium, which results in most DAVFs using the MMA as the feeding artery (9). In our study of DAVFs with MMA involvement, the most common location was the TSS, followed by the tentorium and cavernous sinus, at rates of $31.7,21.2$, and $12.5 \%$, respectively. The reason for this finding is that the branches of the 


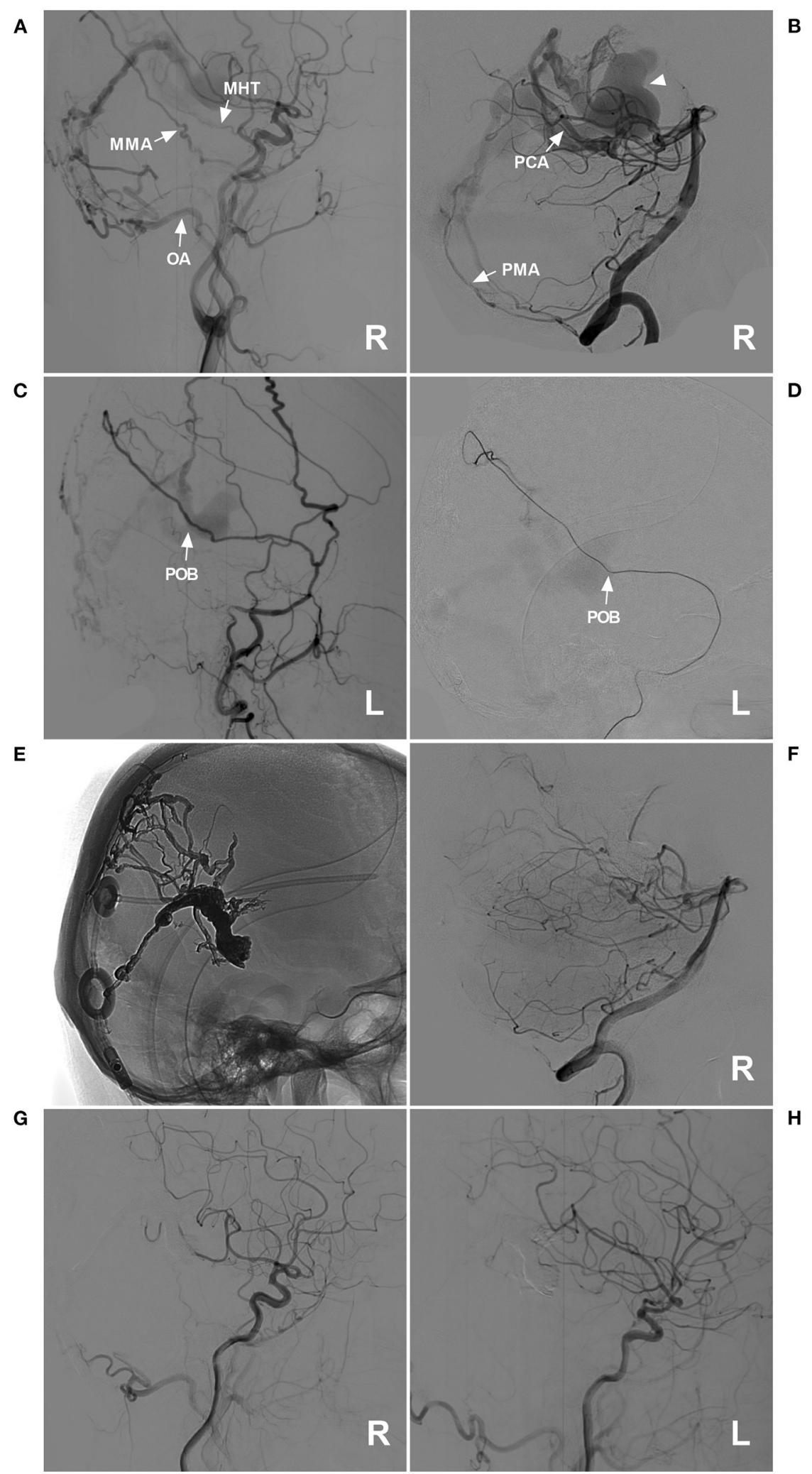

FIGURE 6 | Successful type lla EVT for a DAVF of the posterior falx cerebri. (A) Lateral view DSA of the right carotid artery showed a DAVF at the posterior falx cerebri that was supplied by the MHT, the POB of the MMA, and the OA (arrows). (B) DSA of the right VA showed that the dural branch of the PCA (arrow) and the PMA (arrow) 
FIGURE 6 | supplied the DAVF. The dilated draining vein is indicated by a triangle. (C,D): Lateral view DSA of the left ECA (C) and superselective angiography of the POB (D) showed that the POB was normally developed and straight (arrows). (E) X-ray film showing the Onyx casting and previously established ventriculoperitoneal shunt. (F,H) Images of the right VA (F), right carotid artery (G), and left carotid artery (H) showed that the EVT was complete. DAVF, dural arteriovenous fistula; DSA, digital subtraction angiography; ECA, external carotid artery; EVT, endovascular treatment; L, left; MHT, meningohypophyseal trunk; MMA, middle meningeal artery; OA, occipital artery; PCA, posterior cerebral artery; PMA, posterior meningeal artery; POB, parieto-occipital branch; R, right; VA, vertebral artery.

MMA easily reach these locations, which tend to exhibit DAVFs. For cranial DAVFs, the MMA is the most common source as the feeding artery (10). Certainly, other arteries may be involved, including the OA, PMA, and MHT, which occurred at rates of $47.1,29.8$, and $26.0 \%$, respectively. These arteries tend to supply the DAVF, but this tendency is because DAVFs of the TSS and tentorium were more common in our study.

In general, the symptoms of DAVFs are divided into hemorrhagic and nonhemorrhagic types (10). In our study of 104 DAVFs, $46.2 \%$ of patients had hemorrhagic symptoms at onset. The hemorrhagic onset was primarily from cortical and deep vein drainage (1). The most common pattern of drainage was via multiple cortical veins, with a rate of $33.7 \%$.

The symptoms of DAVFs depended on the pattern of venous drainage, as shown in Figure 1A. Although the arterial blood was diverted to a local vein in some cases, the vein was unruptured, and there were fewer or no symptoms. Figure 2B shows a ruptured venous aneurysm, and this patient presented with hemorrhagic symptoms. Although the veins were not ruptured in Figure 2C, the arterial blood was diverted into the whole brain vein via the vein of Galen with reverse flow. This pattern is called an extensive pseudophlebitic pattern, and the patient presented with cognitive deficiencies (11). The extensive pseudophlebitic pattern occurred at a rate of $4.8 \%$ in our study, and Cognard grade IV accounted for $54.8 \%$ of these cases, which corresponded to the hemorrhagic presentation.

Our study involves an in-depth evaluation of the role of MMA in the transarterial EVT of 98 DAVFs after the exclusion of six diffuse DAVFs. Most of the arteries throughout the entire cranium were recruited in these cases of extensive and diffuse DAVFs, including the bilateral arteries and dural branches of intracranial arteries (Figure 9) and the posterior cerebral artery (the artery of Davidoff and Schechter), superior cerebellar artery (the artery of Wollschlaeger and Wollschlaeger), anterior inferior cerebellar artery and posterior inferior cerebellar artery $(12,13)$.

It is apparent that EVT via these dural branches of the intracranial arteries is difficult and dangerous (14). Therefore, transarterial EVT must depend more on the MMA (15). The common branches in our study included the PSB, POB, petrosal branch, cavernous branch, lateral branch and medial branch (sphenoid) of the anterior division. Under normal conditions, these vessels are slim or invisible, but they become thicker and overdeveloped under the pathological condition of serving as feeding arteries of a DAVF (Figure 2).

We treated DAVFs via the MMA and found that some DAVFs could be completely embolized but that other DAVFs could not, and we wanted to know the underlying reason for this difference. The first path chosen for EVT is very important and key to successful EVT. Therefore, the MMA branch must be evaluated carefully. Our study design considered thickness and tortuosity to be the most influential factors. Therefore, we divided EVT into three types and six subclasses (Figure 3).

Statistical analyses showed that type Ia and IIb EVTs had the lowest complete embolization rates, and type IIa and III EVTs had the highest complete embolization rates. This result means that a thicker and straighter MMA branch is the preferred path. If only types IIa and III EVTs are performed, the success rate may be near 90\%. EVT for DAVFs with MMA involvement generally resulted in a good prognosis, and mRS scores of 0 and 1 were achieved in $78.8 \%$ of patients. However, EVT resulted in complications in $6.7 \%$ of patients.

Complications included facial numbness in two cases, blindness in one case, intraoperative or postoperative intracranial hemorrhage in three cases, and postoperative hydrocephalus in one case. Facial numbness, as a form of cranial nerve damage, resulted from the dangerous anastomosis of the MMA, in which the liquid material enters the feeder to affect the geniculate ganglion (16). In the case of blindness, the liquid material entered the ophthalmic artery via the MMA (17). If the fistula ruptures during EVT under the high pressure of Onyx casting or if too many draining veins are occluded, intracranial hemorrhage may occur. Therefore, EVT must be performed carefully and gently to prevent damage to the draining veins (18). If the draining veins are too thick and near the aqueduct of the midbrain, thrombosis in the draining veins after EVT may cause hydrocephalus (19), and a cerebrospinal fluid shunt is needed.

\section{CONCLUSIONS}

The findings of this study elucidated the features of different EVT classes as defined by the first EVT procedure and the role of the MMA. The delivery of treatment via slim and tortuous MMA branches increased the failure rate of EVT. A thick, straight MMA branch is the optimal path for treatment. EVT for DAVFs with MMA involvement generally results in a good prognosis.

\section{Limitations}

This was a retrospective study, and its conclusions should be interpreted cautiously. As a result of the economic status in rural areas in China, angiographic follow-up data were challenging to obtain, which made it difficult to evaluate the long-term efficacy of EVT. The short-term follow-up in this study was complete, and we performed long-term telephone follow-up in April 2021. Only 50\% of patients had clinical follow-up data, and $20 \%$ of patients had imaging follow-up data. There was an inherent flaw in the measurement of the 


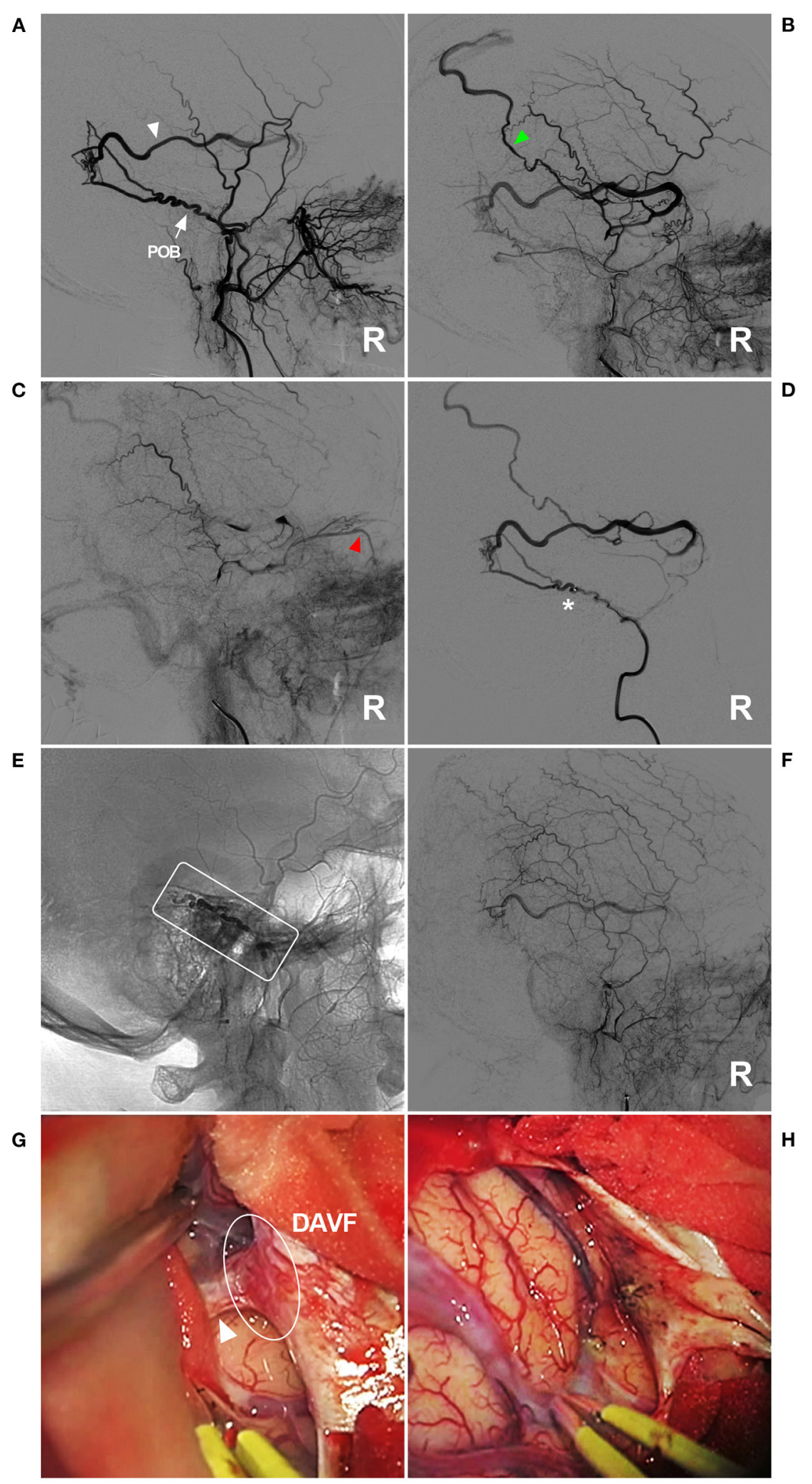

FIGURE 7 | Unsuccessful type Ilb EVT for a DAVF in the temporal region. (A-C) Lateral view ECA DSA showed a DAVF in the temporal region supplied by a normally developed and tortuous POB (arrow) (A). The draining vein behind the Sylvian fissure is clearly shown [white triangle in panel (A), early arterial phase] draining to the 
FIGURE 7 | superior sagittal sinus [green triangle in panel (B), early arterial phase] and the ophthalmic vein [red triangle in panel (C), late arterial phase]. (D) Right MMA angiography showed that the microcatheter could not pass through the tortuous POB (asterisk). (E) X-ray film showing that Onyx (frame) could not reach the point of the fistula. (F) DSA showing the incomplete EVT. (G) Intraoperative imaging showing the DAVF (ellipse) and the draining vein (triangle). (H) The DAVF and draining vein were coagulated and cut. DAVF, dural arteriovenous fistula; DSA, digital subtraction angiography; ECA, external carotid artery; EVT, endovascular treatment; MMA, middle meningeal artery; POB, parieto-occipital branch; $\mathrm{R}$, right.

A

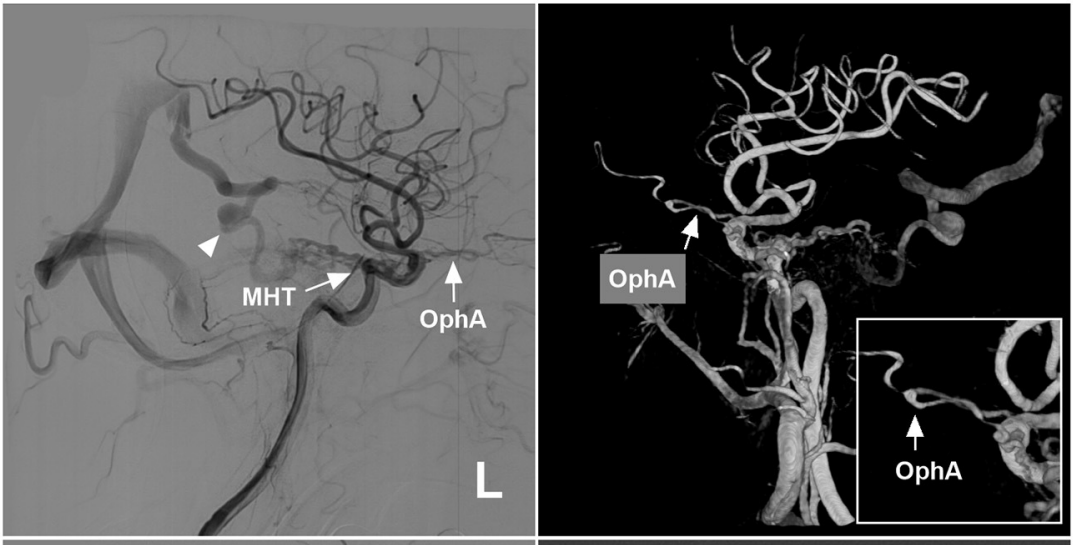

C

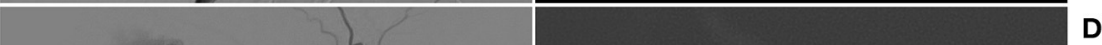

D
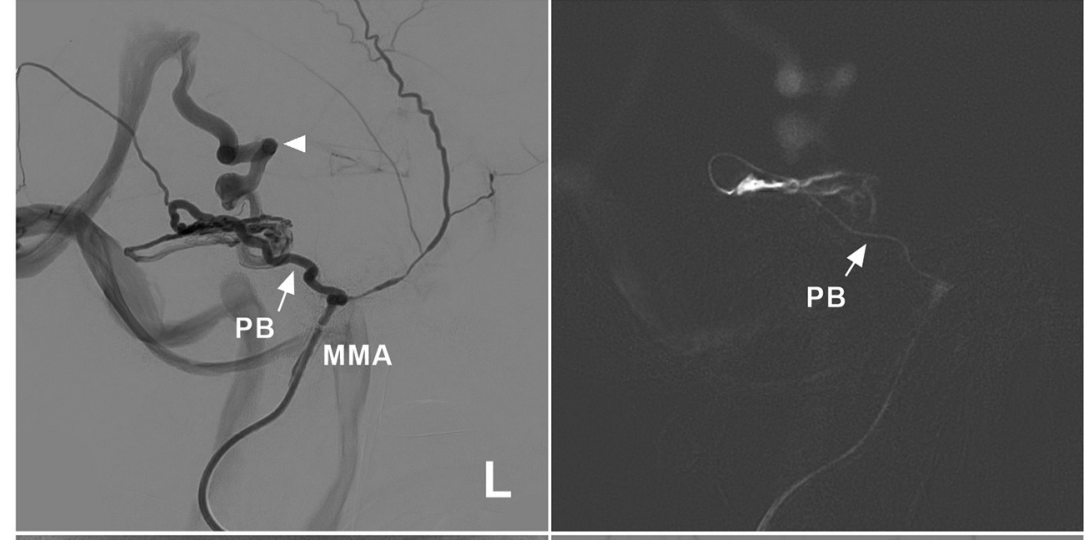

$\mathbf{E}$

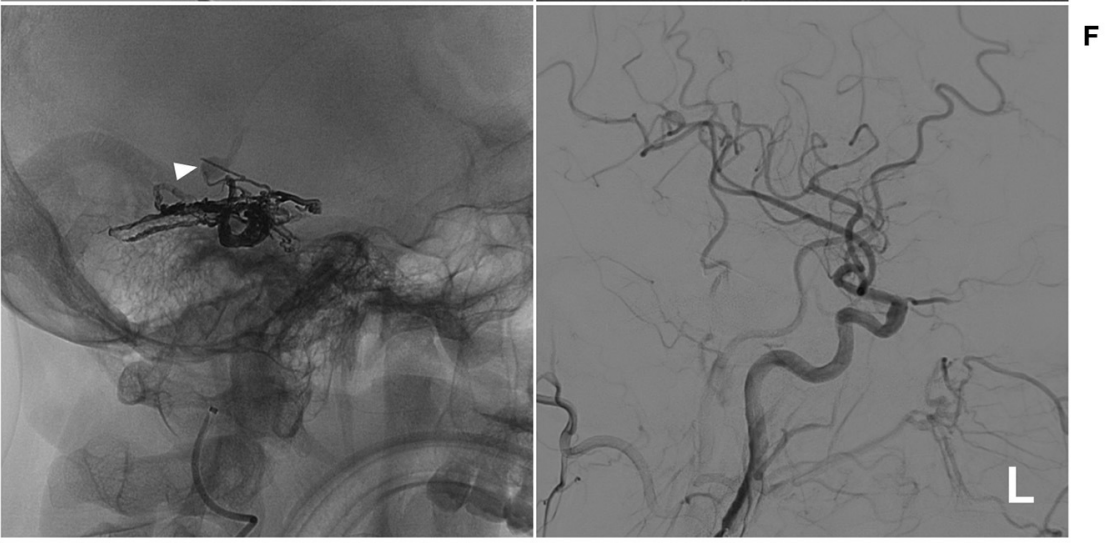

FIGURE 8 | Successful type IIIb EVT for a DAVF of the tentorium. (A) Lateral view DSA of the left carotid artery showed a DAVF supplied by the MHT and OphA (arrows) draining to the vein of Galen (triangle). (B) Three-dimensional DSA showed that the recurrent meningeal branch of the OphA supplied the DAVF (arrow). The inset in the lower right shows the magnified local OphA (arrow). (C) Left MMA angiography showed that the PB of the MMA supplied the DAVF. The triangle indicates deep vein drainage. (D) A roadmap image shows that the microcatheter passed through the PB (arrow) and into the fistula. (E) X-ray film showing the casting Onyx. Onyx penetrated the fistula and sealed the draining vein (triangle). (F) The left carotid artery showed that the EVT was complete. DAVF, dural arteriovenous fistula; DSA, digital subtraction angiography; EVT, endovascular treatment; L, left; MHT, meningohypophyseal trunk; MMA, middle meningeal artery; OphA, ophthalmic artery; $\mathrm{PB}$, petrous branch. 


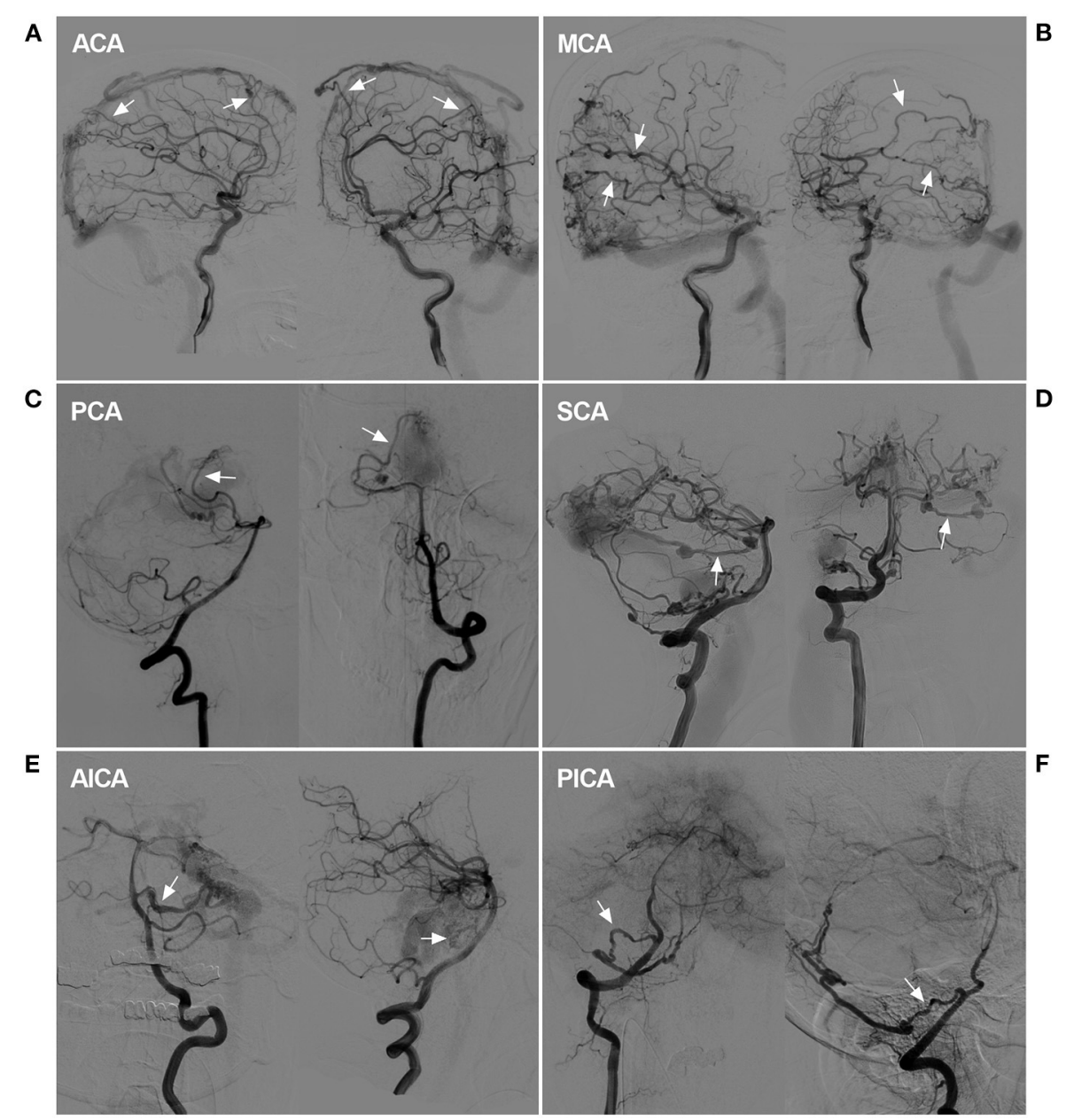

FIGURE 9 | Rare instance of a pial artery serving as the feeder for a DAVF. (A) DSA showed that the ACA sent dural branches (arrows) to supply a SSS DAVF. (B) DSA showed that the MCA sent dural branches (arrows) to supply a SSS DAVF. (C) DSA showed that the PCA (arrows) sent a dural branch to supply a tentorial DAVF. (D) DSA showed that the SCA (arrows) sent a dural branch to supply a TSS DAVF. A flow-related aneurysm may be seen. (E) DSA showed that the AICA (arrows) sent a dural branch to supply a tentorial DAVF. A dissecting aneurysm may be seen. (F) DSA showed that the PICA (arrows) connected to the PMA to supply a tentorial DAVF. ACA, anterior cerebral artery, AICA, anterior inferior cerebellar artery; DAVF, dural arteriovenous fistula; DSA, digital subtraction angiography; MCA, middle cerebral artery, PCA, posterior cerebral artery, PICA, posterior inferior cerebellar artery; PMA, posterior meningeal artery; SCA, superior cerebellar artery; SSS, superior sagittal sinus; TSS, transverse-sigmoid sinus.

MMA diameter because it is impossible to accurately obtain the entire course, and only the origin of the MMA branch from the trunk was measured as the EVT path. Due to the retrospective nature of the study, these data were obtained only for some patients, which reduces the clinical importance of these data. However, our analyses of the arterial path used in the first EVT procedure and the role of the MMA in EVT are of the utmost importance.

\section{DATA AVAILABILITY STATEMENT}

The original contributions presented in the study are included in the article/supplementary material, further inquiries can be directed to the corresponding author/s.

\section{ETHICS STATEMENT}

The studies involving human participants were reviewed and approved by the First Hospital of Jilin University. The patients/participants provided their written informed consent to participate in this study. Written informed consent was obtained from the individual(s) for the publication of any potentially identifiable images or data included in this article.

\section{AUTHOR CONTRIBUTIONS}

JY and KX: contributed to the conception and design of the manuscript and critically revised the manuscript. HS and YW: wrote the manuscript. HS: collected the medical records of the patients. All authors approved the final version of this manuscript. 


\section{REFERENCES}

1. Melo Neto JF, Pelinca da Costa EE, Pinheiro Junior N, Batista AL, Rodesch G, Bracard S, et al. Cerebral venous drainage in patients with dural arteriovenous fistulas: correlation with clinical presentation. J Neurosurg. (2020) 1-9. doi: 10.3171/2020.6.JNS20922. [Epub ahead of print].

2. Baharvahdat H, Ooi YC, Kim WJ, Mowla A, Coon AL, Colby GP. Updates in the management of cranial dural arteriovenous fistula. Stroke Vasc Neurol. (2020) 5:50-8. doi: 10.1136/svn-2019-000269

3. Qureshi AM, Bhatia K, Kostynskyy A, Krings T. Clinical and angioarchitectural features of ruptured dural arterio-venous fistulas. World Neurosurg. (2021) 147:e476-81. doi: 10.1016/j.wneu.2020.12.091

4. Hiramatsu M, Sugiu K, Hishikawa T, Nishihiro S, Kidani N, Takahashi Y, et al. Results of 1940 embolizations for dural arteriovenous fistulas: Japanese Registry of Neuroendovascular Therapy (JR-NET3). J Neurosurg. (2019) 18. doi: 10.3171/2019.4.JNS183458

5. Xu K, Ji T, Li C, Yu J. Current status of endovascular treatment for dural arteriovenous fistulae in the anterior cranial fossa: a systematic literature review. Int J Med Sci. (2019) 16:203-11. doi: 10.7150/ijm s.29637

6. Padilha IG, Pacheco FT, Araujo AIR, Nunes RH, Baccin CE, Conti MLM, et al. Tips and tricks in the diagnosis of intracranial dural arteriovenous fistulas: a pictorial review. J Neuroradiol. (2020) 47:36981. doi: 10.1016/j.neurad.2019.06.004

7. Hou K, Li G, Luan T, Xu K, Yu J. Endovascular treatment of the cavernous sinus dural arteriovenous fistula: current status and considerations. Int J Med Sci. (2020) 17:1121-30. doi: 10.7150/ijms.45210

8. Griessenauer CJ, He L, Salem M, Chua MH, Ogilvy CS, Thomas AJ. Middle meningeal artery: gateway for effective transarterial Onyx embolization of dural arteriovenous fistulas. Clin Anat. (2016) 29:71828. doi: 10.1002/ca.22733

9. Martins C, Yasuda A, Campero A, Ulm AJ, Tanriover N, Rhoton A Jr. Microsurgical anatomy of the dural arteries. Neurosurgery. (2005) 56:211-51; discussion 51. doi: 10.1227/01.NEU.0000144823.94402.3D

10. Sugiyama T, Nakayama N, Ushikoshi S, Kazumata K, Okamoto M, Ito M, et al. Complication rate, cure rate, and long-term outcomes of microsurgery for intracranial dural arteriovenous fistulae: a multicenter series and systematic review. Neurosurg Rev. (2021) 44:435-50. doi: 10.1007/s10143-01901232-y

11. Brinjikji W, Cloft HJ, Lanzino G. Clinical presentation and imaging findings of patients with dural arteriovenous fistulas with an angiographic pseudophlebitic pattern. AJNR Am J Neuroradiol. (2020) 41:2285-91. doi: 10.3174/ajnr.A6811

12. Tong $\mathrm{D}$, Chen $\mathrm{X}, \mathrm{Lv} \mathrm{X}, \mathrm{Li} \mathrm{K}, \mathrm{Xu} \mathrm{K}, \mathrm{Yu}$ J. Current status of endovascular treatment for dural arteriovenous fistulae in the tentorial middle region: a literature review. Acta Neurol Belg. (2019) 119:514. doi: 10.1007/s13760-018-1044-3

13. Bhatia KD, Kortman H, Walchli T, Radovanovic I, Pereira VM, Krings T. Artery of Davidoff and Schechter supply in dural arteriovenous fistulas. AJNR Am J Neuroradiol. (2020) 41:300-4. doi: 10.3174/ajnr.A6380

14. Brinjikji W, Cloft HJ, Lanzino G. Clinical, angiographic, and treatment characteristics of cranial dural arteriovenous fistulas with pial arterial supply. J Neurointerv Surg. (2021) 13:331-5. doi: 10.1136/neurintsurg-2020-016374

15. Oh SH, Choi JH, Kim BS, Lee KS, Shin YS. Treatment outcomes according to various treatment modalities for intracranial dural arteriovenous fistulas in the onyx era: a 10-year single-center experience. World Neurosurg. (2019) 126:e825-e34. doi: 10.1016/j.wneu.2019.02.173

16. Hou K, Lv X, Qu L, Guo Y, Xu K, Yu J. Endovascular treatment for dural arteriovenous fistulas in the petroclival region. Int J Med Sci. (2020) 17:302030. doi: 10.7150/ijms.47365

17. Hou K, Wu W, Liu Y, Qu L, Xu B, Yu J. Role of the ophthalmic artery in the endovascular treatment for intracranial vascular diseases. Acta Neurol Belg. (2021) 121:321-30. doi: 10.1007/s13760-020-01576-Z

18. Li Y, Chen SH, Guniganti R, Kansagra AP, Piccirillo JF, Chen CJ, et al. Onyx embolization for dural arteriovenous fistulas: a multi-institutional study. J Neurointerv Surg. (2021). doi: 10.1136/neurintsurg-2020-017109. [Epub ahead of print].

19. Nakahara Y, Ogata A, Takase Y, Maeda K, Okamoto H, Matsushima T, et al. Treatment of dural arteriovenous fistula presenting as typical symptoms of hydrocephalus caused by venous congestion: case report. Neurol Med Chir (Tokyo). (2011) 51:229-32. doi: 10.2176/nmc.51.229

Conflict of Interest: The authors declare that the research was conducted in the absence of any commercial or financial relationships that could be construed as a potential conflict of interest.

Copyright (c) $2021 \mathrm{Su}, \mathrm{Xu}$, Wang and Yu. This is an open-access article distributed under the terms of the Creative Commons Attribution License (CC BY). The use, distribution or reproduction in other forums is permitted, provided the original author(s) and the copyright owner(s) are credited and that the original publication in this journal is cited, in accordance with accepted academic practice. No use, distribution or reproduction is permitted which does not comply with these terms. 